\title{
A unique common coupled fixed point theorem for four maps under $\psi-\phi$ contractive condition in partial metric spaces
}

K.P.R.RAO
Department of Mathematics,
Nagarjuna Nagar522 510,
Acharya Nagarjuna Univertsity
Guntur District,Andhra
Pradesh,India
kprrao2004@yahoo.com

\author{
G.N.V.KISHORE \\ Department of Mathematics, \\ Swarnandhra Institute of \\ Engineering and Technology, \\ West Godavari District, Andhra \\ Pradesh, India \\ kishore.apr2@gmail.com
}

\author{
NGuYen VAn LuONG \\ Department of Natural \\ Sciences, \\ Hong Duc University, \\ Thanh Hoa, Viet Nam \\ luonghdu@gmail.com
}

\begin{abstract}
In this paper, we obtain a unique coupled common fixed point theorem for four maps in partial metric spaces.

\section{RESUMEN}

En este artículo obtenemos un teorema del punto fijo clásico acoplado único para cuatro aplicaciones en espacios métricos parciales.
\end{abstract}

Keywords and Phrases: Partial metric, weakly compatible maps, complete space.

2010 AMS Mathematics Subject Classification: 54H25, 47H10. 


\section{Introduction and Preliminaries}

The notion of partial metric space was introduced by S.G.Matthews [13] as a part of the study of denotational semantics of data flow networks. In fact, it is widely recognized that partial metric spaces play an important role in constructing models in the theory of computation ([6-10, 14-16], etc).

S.G.Matthews [13], Sandra Oltra and Oscar Valero[11] and Salvador Romaguera [12] and I.Altun, Ferhan Sola, Hakan Simsek [1], T. Abdeljawad, E. Karapinar, K. Tas [3], E. Karapinar, I.M. Erhan [5] proved fixed point theorems in partial metric spaces for a single map and a pair of maps.

Regarding the concept of coupled fixed points introduced by Bhaskar and Lakshmikantham [17], in [4], Aydi proved some coupled fixed point theorems for the mappings satisfying contractive conditions in partial metric spaces. In this paper, we obtain a unique common coupled fixed point theorem for four self mappings satisfying a $\psi-\phi$ contractive condition in partial metric spaces. Our result is inspired by the results of Luong and Thuan [18].

First we recall some definitions and lemmas of partial metric spaces.

Definition 1.1. [13]. A partial metric on a nonempty set $X$ is a function $p: X \times X \rightarrow R^{+}$such that for all $x, y, z \in X$ :

$\left(p_{1}\right) \quad x=y \Leftrightarrow p(x, x)=p(x, y)=p(y, y)$,

$\left(p_{2}\right) \quad p(x, x) \leq p(x, y), p(y, y) \leq p(x, y)$,

$\left(p_{3}\right) \quad p(x, y)=p(y, x)$,

$\left(p_{4}\right) \quad p(x, y) \leq p(x, z)+p(z, y)-p(z, z)$.

$(X, p)$ is called a partial metric space.

It is clear that $p(x, y)=0$ implies $x=y$ from $\left(p_{1}\right)$ and $\left(p_{2}\right)$.

But if $x=y, p(x, y)$ may not be zero. A basic example of a partial metric space is the pair $\left(R^{+}, p\right)$, where $p(x, y)=\max \{x, y\}$ for all $x, y \in R^{+}$.

Each partial metric $p$ on $X$ generates $\tau_{0}$ topology $\tau_{p}$ on $X$ which has a base the family of open $p$ - balls $\left\{B_{p}(x, \varepsilon) \mid x \in X, \varepsilon>0\right\}$ for all $x \in X$ and $\varepsilon>0$, where $B_{p}(x, \varepsilon)=\{y \in X / p(x, y)<p(x, x)+\varepsilon\}$ for all $x \in X$ and $\varepsilon>0$.

If $p$ is a partial metric on $X$, then the function $p^{s}: X \times X \rightarrow R^{+}$given by $p^{s}(x, y)=2 p(x, y)-$ $p(x, x)-p(y, y)$ is a metric on $X$.

Definition 1.2. [13]. Let (X, p) be a partial metric space.

(i) A sequence $\left\{x_{n}\right\}$ in $(X, p)$ is said to converge to a point $x \in X$ if and only if $p(x, x)=$ $\lim _{n \rightarrow \infty} p\left(x, x_{n}\right)$.

(ii) A sequence $\left\{x_{n}\right\}$ in $(X, p)$ is said to be Cauchy sequence if $\lim _{n, m \rightarrow \infty} p\left(x_{n}, x_{m}\right)$ exists and is finite

(iii) $(X, p)$ is said to be complete if every Cauchy sequence $\left\{x_{n}\right\}$ in $X$ converges, w.r.to $\tau_{p}$, to a point $x \in X$ such that $p(x, x)=\lim _{n, m \rightarrow \infty} p\left(x_{n}, x_{m}\right)$. 
Lemma 1.1. [13]. Let $(X, p)$ be a partial metric space.

(a) $\left\{x_{n}\right\}$ is a Cauchy sequence in $(X, p)$ if and only if it is a Cauchy sequence in the metric space $\left(X, p^{s}\right)$.

(b) $(X, p)$ is complete if and only if the metric space $\left(X, p^{s}\right)$ is complete.

Furthermore, $\lim _{n \rightarrow \infty} p^{s}\left(x_{n}, x\right)=0$ if and only if

$p(x, x)=\lim _{n \rightarrow \infty} p\left(x_{n}, x\right)=\lim _{n, m \rightarrow \infty} p\left(x_{n}, x_{m}\right)$.

Remark 1.2. Let $(X, p)$ be a partial metric space. If $\left\{x_{n}\right\}$ converges to $x$ in $(X, p)$, then

$$
\lim _{n \rightarrow \infty} p\left(x_{n}, y\right) \leq p(x, y), \quad \forall y \in X .
$$

Proof. Since $\left\{x_{n}\right\}$ converges to $x$ we have $p(x, x)=\lim _{n \rightarrow \infty} p\left(x_{n}, x\right)$.

Now $p\left(x_{n}, y\right) \leq p\left(x_{n}, x\right)+p(x, y)-p(x, x)$.

Letting $\mathrm{n} \rightarrow \infty$,

$$
\lim _{n \rightarrow \infty} p\left(x_{n}, y\right) \leq \lim _{n \rightarrow \infty} p\left(x_{n}, x\right)+p(x, y)-p(x, x) .
$$

Thus $\lim _{n \rightarrow \infty} p\left(x_{n}, y\right) \leq p(x, y)$.

Definition 1.3. [17]. An element $(x, y) \in X \times X$ is called a coupled fixed point of mapping $F$ : $X \times X \rightarrow X$ if $x=F(x, y)$ and $y=F(y, x)$.

Definition 1.4. [2]. An element $(x, y) \in X \times X$ is called

$\left(g_{1}\right)$ a coupled coincident point of mappings $F: X \times X \rightarrow X$ and $f: X \rightarrow X$ if $f \chi=F(x, y)$ and $f y=F(y, x)$.

$\left(g_{2}\right)$ a common coupled fixed point of mappings $F: X \times X \rightarrow X$ and $f: X \rightarrow X$ if $x=f x=F(x, y)$ and $y=f y=F(y, x)$.

Definition 1.5. [2]. The mappings $F: X \times X \rightarrow X$ and $f: X \rightarrow X$ are called $w$ - compatible if $f(F(x, y))=F(f x, f y)$ and $f(F(y, x))=F(f y, f x)$ whenever $f x=F(x, y)$ and $f y=F(y, x)$.

Using concept of coupled fixed points, Luong and Thuan in [18] proved some coupled fixed point theorems for a mapping $\mathrm{F}: \mathrm{X} \times \mathrm{X} \rightarrow \mathrm{X}$ satisfying the following contractive condition in the partially ordered metric spaces $(X, d, \leq)$

$$
\psi(d(F(x, y), F(u, v))) \leq \frac{1}{2} \psi(d(x, u)+d(y, v))-\phi\left(\frac{d(x, u)+d(y, v)}{2}\right)
$$

for all $x, y, u, v \in X$ with $x \geq u$ and $y \leq v$, with $\phi \in \Phi$ and $\psi \in \Psi$, where $\Psi$ denotes the set of all functions $\psi:[0, \infty) \rightarrow[0, \infty)$ satisfying

$\left(\psi_{1}\right) \psi$ is continuous and non-decreasing,

$\left(\psi_{2}\right) \psi(t)=0$ if and only if $t=0$,

$\left(\psi_{3}\right) \psi(t+s) \leq \psi(t)+\psi(s)$, for all $t, s \in[0, \infty)$,

while $\Phi$ denotes the set of all functions $\phi:[0, \infty) \rightarrow[0, \infty)$ satisfying

$\left(\phi_{1}\right) \lim _{t \rightarrow r} \phi(t)>0$ for all $r>0$. 
$\left(\phi_{2}\right) \lim _{\mathrm{t} \rightarrow 0+} \phi(\mathrm{t})=0$.

From $\left(\phi_{1}\right)$, it is clear that $\phi(t)>0$ for all $t>0$.

Now we prove our main result.

\section{Main Result}

Theorem 1. Let $(X, p)$ be a partial metric space and let $f, g: X \rightarrow X$ and $F, G: X \times X \rightarrow X$ be such that

(i) For all $x, y, u, v \in X$,

$$
\psi(p(F(x, y), G(u, v))) \leq \frac{1}{2} \psi(p(f x, g u)+p(f y, g v))-\phi(p(f x, g u)+p(f y, g v)),
$$

where $\psi \in \Psi$ and $\phi \in \Phi$,

(ii) $F(X \times X) \subseteq g(X), G(X \times X) \subseteq f(X)$,

(iii) either $f(X)$ or $g(X)$ is a complete subspace of $X$ and

(iv) the pairs $(F, f)$ and $(G, g)$ are $w$ - compatible.

Then $F, G, f$ and $g$ have a unique common coupled fixed point in $X \times X$. Moreover, the common coupled fixed point of $F, G, f$ and $g$ have the form $(u, u)$.

Proof. Let $x_{0}, y_{0}$ be arbitrary points in $X$.

From(ii), there exist sequences $\left\{x_{n}\right\},\left\{y_{n}\right\},\left\{z_{n}\right\}$ and $\left\{w_{n}\right\}$ in $X$ such that

$$
\begin{gathered}
F\left(x_{2 n}, y_{2 n}\right)=g x_{2 n+1}=z_{2 n}, \\
F\left(y_{2 n}, x_{2 n}\right)=g y_{2 n+1}=w_{2 n}, \\
G\left(x_{2 n+1}, y_{2 n+1}\right)=f x_{2 n+2}=z_{2 n+1}
\end{gathered}
$$

and

$$
G\left(y_{2 n+1}, x_{2 n+1}\right)=f y_{2 n+2}=w_{2 n+1},
$$

$\mathrm{n}=0,1,2, \ldots \ldots$.

We have

$$
\begin{aligned}
\psi\left(p\left(z_{2 n+1}, z_{2 n}\right)\right)= & \psi\left(p\left(F\left(x_{2 n}, y_{2 n}\right), G\left(x_{2 n+1}, y_{2 n+1}\right)\right)\right. \\
\leq & \frac{1}{2} \psi\left(p\left(z_{2 n}, z_{2 n-1}\right)+p\left(w_{2 n}, w_{2 n-1}\right)\right) \\
& -\phi\left(p\left(z_{2 n}, z_{2 n-1}\right)+p\left(w_{2 n}, w_{2 n-1}\right)\right)
\end{aligned}
$$

Similarly,

$$
\begin{aligned}
\psi\left(p\left(w_{2 n+1}, w_{2 n}\right)\right) \leq & \frac{1}{2} \psi\left(p\left(z_{2 n}, z_{2 n-1}\right)+p\left(w_{2 n}, w_{2 n-1}\right)\right) \\
& -\phi\left(p\left(z_{2 n}, z_{2 n-1}\right)+p\left(w_{2 n}, w_{2 n-1}\right)\right)
\end{aligned}
$$


From $(2.1),(2.2)$ and $\left(\psi_{3}\right)$, we have

$$
\begin{aligned}
\psi\left(p\left(z_{2 n+1}, z_{2 n}\right)+p\left(w_{2 n+1}, w_{2 n}\right)\right) \leq & \psi\left(p\left(z_{2 n+1}, z_{2 n}\right)\right)+\psi\left(p\left(w_{2 n+1}, w_{2 n}\right)\right) \\
\leq & \psi\left(p\left(z_{2 n}, z_{2 n-1}\right)+p\left(w_{2 n}, w_{2 n-1}\right)\right) \\
& -2 \phi\left(p\left(z_{2 n}, z_{2 n-1}\right)+p\left(w_{2 n}, w_{2 n-1}\right)\right) \\
\leq & \psi\left(p\left(z_{2 n}, z_{2 n-1}\right)+p\left(w_{2 n}, w_{2 n-1}\right)\right) .
\end{aligned}
$$

Since $\psi$ is non - decreasing, we have

$$
p\left(z_{2 n+1}, z_{2 n}\right)+p\left(w_{2 n+1}, w_{2 n}\right) \leq p\left(z_{2 n}, z_{2 n-1}\right)+p\left(w_{2 n}, w_{2 n-1}\right) .
$$

Similarly, we can show that

$$
p\left(z_{2 n}, z_{2 n-1}\right)+p\left(w_{2 n}, w_{2 n-1}\right) \leq p\left(z_{2 n-1}, z_{2 n-2}\right)+p\left(w_{2 n-1}, w_{2 n-2}\right) .
$$

Thus

$$
p\left(z_{n+1}, z_{n}\right)+p\left(w_{n+1}, w_{n}\right) \leq p\left(z_{n}, z_{n-1}\right)+p\left(w_{n}, w_{n-1}\right) .
$$

Put $\delta_{n}=p\left(z_{n+1}, z_{n}\right)+p\left(w_{n+1}, w_{n}\right)$. Then we have

$$
\delta_{\mathrm{n}} \leq \delta_{\mathrm{n}-1}, \mathrm{n}=1,2,3, \ldots
$$

Thus $\left\{\delta_{n}\right\}$ is a non - increasing sequence of non- negitive real numbers and must converge to a real number, say, $\delta \geq 0$.

Suppose $\delta>0$.

Letting $n \rightarrow \infty$ in (2.3) and using the properties of $\psi$ and $\phi$, we get

$$
\psi(\delta) \leq \psi(\delta)-2 \lim _{\delta_{2 n} \rightarrow \delta} \phi\left(\delta_{2 n}\right)<\psi(\delta)
$$

which is a contradiction. Hence $\delta=0$.

Thus

$$
\lim _{n \rightarrow \infty}\left[p\left(z_{n+1}, z_{n}\right)+p\left(w_{n+1}, w_{n}\right)\right]=0
$$

Hence from $\left(\mathrm{p}_{2}\right)$,

$$
\lim _{n \rightarrow \infty}\left[p\left(z_{n}, z_{n}\right)+p\left(w_{n}, w_{n}\right)\right]=0
$$

From (2.4) and (2.5) we have that

$$
\lim _{n \rightarrow \infty} p^{s}\left(z_{n+1}, z_{n}\right)=0
$$

and

$$
\lim _{n \rightarrow \infty} p^{s}\left(w_{n+1}, w_{n}\right)=0
$$

Now we prove that $\left\{z_{2 n}\right\}$ and $\left\{w_{2 n}\right\}$ are Cauchy sequences.

On contrary, suppose that $\left\{z_{2 n}\right\}$ or $\left\{w_{2 n}\right\}$ is not Cauchy. This implies that $\mathrm{p}^{s}\left(z_{2 m}, z_{2 n}\right) \not \rightarrow 0$ or 
$p^{s}\left(w_{2 m}, w_{2 n}\right) \not \rightarrow 0$ as $n, m \rightarrow \infty$

Consequently

$$
\max \left\{p^{s}\left(z_{2 m}, z_{2 n}\right), p^{s}\left(w_{2 m}, w_{2 n}\right)\right\} \not \rightarrow 0 \text { as } n, m \rightarrow \infty .
$$

Then there exist an $\epsilon>0$ and monotone increasing sequences of natural numbers $\left\{2 m_{k}\right\}$ and $\left\{2 n_{k}\right\}$ such that $n_{k}>m_{k}$,

$$
\max \left\{p^{s}\left(z_{2 m_{k}}, z_{2 n_{k}}\right), p^{s}\left(w_{2 m_{k}}, w_{2 n_{k}}\right)\right\} \geq \epsilon
$$

and

$$
\max \left\{p^{s}\left(z_{2 m_{k}}, z_{2 n_{k}-2}\right), p^{s}\left(w_{2 m}, w_{2 n_{k}-2}\right)\right\}<\epsilon .
$$

From (2.8) and (2.9), we have

$$
\begin{aligned}
\epsilon \leq & \max \left\{p^{s}\left(z_{2 m_{k}}, z_{2 n_{k}}\right), p^{s}\left(w_{2 m_{k}}, w_{2 n_{k}}\right)\right\} \\
\leq & \max \left\{p^{s}\left(z_{2 m_{k}}, z_{2 n_{k}-2}\right), p^{s}\left(w_{2 m_{k}}, w_{2 n_{k}-2}\right)\right\} \\
& +\max \left\{p^{s}\left(z_{2 n_{k}-2}, z_{2 n_{k}-1}\right), p^{s}\left(w_{2 n_{k}-2}, w_{2 n_{k}-1}\right)\right\} \\
& +\max \left\{p^{s}\left(z_{2 n_{k}-1}, z_{2 n_{k}}\right), p^{s}\left(w_{2 n_{k}-1}, w_{2 n_{k}}\right)\right\} \\
< & \epsilon+\max \left\{p^{s}\left(z_{2 n_{k}-2}, z_{2 n_{k}-1}\right), p^{s}\left(w_{2 n_{k}-2}, w_{2 n_{k}-1}\right)\right\} \\
& +\max \left\{p^{s}\left(z_{2 n_{k}-1}, z_{2 n_{k}}\right), p^{s}\left(w_{2 n_{k}-1}, w_{2 n_{k}}\right)\right\} .
\end{aligned}
$$

Letting $k \rightarrow \infty$ and using (2.6) and (2.7) we have

$$
\lim _{k \rightarrow \infty} \max \left\{p^{s}\left(z_{2 m_{k}}, z_{2 n_{k}}\right), p^{s}\left(w_{2 m_{k}}, w_{2 n_{k}}\right)\right\}=\epsilon
$$

Also,

$$
\begin{aligned}
\epsilon \leq & \max \left\{p^{s}\left(z_{2 m_{k}}, z_{2 n_{k}}\right), p^{s}\left(w_{2 m_{k}}, w_{2 n_{k}}\right)\right\} \\
\leq & \max \left\{p^{s}\left(z_{2 m_{k}}, z_{2 m_{k}-1}\right), p^{s}\left(w_{2 m_{k}}, w_{2 m_{k}-1}\right)\right\} \\
& +\max \left\{p^{s}\left(z_{2 m_{k}-1}, z_{2 n_{k}}\right), p^{s}\left(w_{2 m_{k}-1}, w_{2 n_{k}}\right)\right\} \\
\leq & \max \left\{p^{s}\left(z_{2 m_{k}}, z_{2 m_{k}-1}\right), p^{s}\left(w_{2 m_{k}}, w_{2 m_{k}-1}\right)\right\} \\
& +\max \left\{p^{s}\left(z_{2 m_{k}-1}, z_{2 m_{k}}\right), p^{s}\left(w_{2 m_{k}-1}, w_{2 m_{k}}\right)\right\} \\
& +\max \left\{p^{s}\left(z_{2 m_{k}}, z_{2 n_{k}}\right), p^{s}\left(w_{2 m_{k}}, w_{2 n_{k}}\right)\right\} \\
= & 2 \max \left\{p^{s}\left(z_{2 m_{k}}, z_{2 m_{k}-1}\right), p^{s}\left(w_{2 m_{k}}, w_{2 m_{k}-1}\right)\right\} \\
& +\max \left\{p^{s}\left(z_{2 m_{k}}, z_{2 n_{k}}\right), p^{s}\left(w_{2 m_{k}}, w_{2 n_{k}}\right)\right\} .
\end{aligned}
$$

Letting $k \rightarrow \infty$ and using (2.6), (2.7), (2.10) and (2.11), we have

$$
\lim _{k \rightarrow \infty} \max \left\{p^{s}\left(z_{2 m_{k}-1}, z_{2 n_{k}}\right), p^{s}\left(w_{2 m_{k}-1}, w_{2 n_{k}}\right)\right\}=\epsilon
$$

On other hand we have

$$
\begin{aligned}
\max \left\{p^{s}\left(z_{2 m_{k}}, z_{2 n_{k}}\right), p^{s}\left(w_{2 m_{k}}, w_{2 n_{k}}\right)\right\} \leq \max & \left\{p^{s}\left(z_{2 m_{k}}, z_{2 n_{k}+1}\right), p^{s}\left(w_{2 m_{k}}, w_{2 n_{k}+1}\right)\right\} \\
+ & \max \left\{p^{s}\left(z_{2 n_{k}+1}, z_{2 n_{k}}\right), p^{s}\left(w_{2 n_{k}+1}, w_{2 n_{k}}\right)\right\}
\end{aligned}
$$


Letting $k \rightarrow \infty$ and using (2.5),(2.6) and (2.7), we have

$$
\begin{aligned}
\epsilon & \leq \lim _{k \rightarrow \infty} \max \left\{p^{s}\left(z_{2 m_{k}}, z_{2 n_{k}+1}\right), p^{s}\left(w_{2 m_{k}}, w_{2 n_{k}+1}\right)\right\}+0 \\
& \leq \lim _{k \rightarrow \infty} \max \left\{\begin{array}{c}
2 p\left(z_{2 m_{k}}, z_{2 n_{k}+1}\right)-p\left(z_{2 m_{k}}, z_{2 m_{k}}\right)-p\left(z_{2 n_{k}+1}, z_{2 n_{k}+1}\right), \\
2 p\left(w_{2 m_{k}}, w_{2 n_{k}+1}\right)-p\left(w_{2 m_{k}}, w_{2 m_{k}}\right)-p\left(w_{2 n_{k}+1}, w_{2 n_{k}+1}\right)
\end{array}\right\} \\
& \leq 2 \lim _{k \rightarrow \infty} \max \left\{p\left(z_{2 m_{k}}, z_{2 n_{k}+1}\right), p\left(w_{2 m_{k}}, w_{2 n_{k}+1}\right)\right\}
\end{aligned}
$$

Thus,

$$
\frac{\epsilon}{2} \leq \lim _{k \rightarrow \infty} \max \left\{\mathrm{p}\left(z_{2 \mathrm{~m}_{\mathrm{k}}}, z_{2 \mathrm{n}_{\mathrm{k}}+1}\right), \mathrm{p}\left(\boldsymbol{w}_{2 \mathrm{~m}_{\mathrm{k}}}, w_{2 \mathrm{n}_{\mathrm{k}}+1}\right)\right\}
$$

By the properties of $\psi$

$$
\begin{aligned}
\psi\left(\frac{\epsilon}{2}\right) & \leq \psi\left(\lim _{k \rightarrow \infty} \max \left\{p\left(z_{2 m_{k}}, z_{2 n_{k}+1}\right), p\left(w_{2 m_{k}}, w_{2 n_{k}+1}\right)\right\}\right) \\
& =\lim _{k \rightarrow \infty} \max \left\{\psi\left(p\left(z_{2 m_{k}}, z_{2 n_{k}+1}\right)\right), \psi\left(p\left(w_{2 m_{k}}, w_{2 n_{k}+1}\right)\right)\right\}
\end{aligned}
$$

Now

$$
\begin{aligned}
\psi\left(p\left(z_{2 m_{k}}, z_{2 n_{k}+1}\right)\right)= & \psi\left(p\left(F\left(x_{2 m_{k}}, y_{2 m_{k}}\right), G\left(x_{2 n_{k}+1}, y_{2 n_{k}+1}\right)\right)\right) \\
\leq & \frac{1}{2} \psi\left(p\left(z_{2 m_{k}-1}, z_{2 n_{k}}\right)+p\left(w_{2 m_{k}-1}, w_{2 n_{k}}\right)\right) \\
& -\phi\left(p\left(z_{2 m_{k}-1}, z_{2 n_{k}}\right)+p\left(w_{2 m_{k}-1}, w_{2 n_{k}}\right)\right) \\
\leq & \frac{1}{2}\left[\psi\left(p\left(z_{2 m_{k}-1}, z_{2 n_{k}}\right)\right)+\psi\left(p\left(w_{2 m_{k}-1}, w_{2 n_{k}}\right)\right)\right] \\
& -\phi\left(p\left(z_{2 m_{k}-1}, z_{2 n_{k}}\right)+p\left(w_{2 m_{k}-1}, w_{2 n_{k}}\right)\right) \\
\leq & \max \left\{\psi\left(p\left(z_{2 m_{k}-1}, z_{2 n_{k}}\right)\right), \psi\left(p\left(w_{2 m_{k}-1}, w_{2 n_{k}}\right)\right)\right\} \\
& -\phi\left(p\left(z_{2 m_{k}-1}, z_{2 n_{k}}\right)+p\left(w_{2 m_{k}-1}, w_{2 n_{k}}\right)\right) \\
= & \psi\left(\max \left\{p\left(z_{2 m_{k}-1}, z_{2 n_{k}}\right), p\left(w_{2 m_{k}-1}, w_{2 n_{k}}\right)\right\}\right) \\
& -\phi\left(p\left(z_{2 m_{k}-1}, z_{2 n_{k}}\right)+p\left(w_{2 m_{k}-1}, w_{2 n_{k}}\right)\right)
\end{aligned}
$$

Similarly

$$
\begin{aligned}
\psi\left(p\left(w_{2 m_{k}}, w_{2 n_{k}+1}\right)\right) \leq & \psi\left(\max \left\{p\left(z_{2 m_{k}-1}, z_{2 n_{k}}\right), p\left(w_{2 m_{k}-1}, w_{2 n_{k}}\right)\right\}\right) \\
& -\phi\left(p\left(z_{2 m_{k}-1}, z_{2 n_{k}}\right)+p\left(w_{2 m_{k}-1}, w_{2 n_{k}}\right)\right) .
\end{aligned}
$$


Hence from $(2.13),(2.5)$ and $(2.12)$, we have

$$
\begin{aligned}
& \psi\left(\frac{\epsilon}{2}\right) \leq \lim _{k \rightarrow \infty}\left\{\begin{array}{c}
\psi\left(\max \left\{p\left(z_{2 m_{k}-1}, z_{2 n_{k}}\right), p\left(w_{2 m_{k}-1}, w_{2 n_{k}}\right)\right\}\right) \\
-\phi\left(p\left(z_{2 m_{k}-1}, z_{2 n_{k}}\right)+p\left(w_{2 m_{k}-1}, w_{2 n_{k}}\right)\right)
\end{array}\right\} \\
& \leq \lim _{k \rightarrow \infty} \psi\left[\max \left\{\begin{array}{c}
\frac{1}{2}\left(\begin{array}{c}
p^{s}\left(z_{2 m_{k}-1}, z_{2 n_{k}}\right)+p\left(z_{2 m_{k}-1}, z_{2 m_{k}-1}\right) \\
+p\left(z_{2 n_{k}}, z_{2 n_{k}}\right)
\end{array}\right), \\
\frac{1}{2}\left(\begin{array}{c}
p^{s}\left(w_{2 m_{k}-1}, w_{2 n_{k}}\right)+p\left(w_{2 m_{k}-1}, w_{2 m_{k}-1}\right) \\
+p\left(w_{2 n_{k}}, w_{2 n_{k}}\right)
\end{array}\right)
\end{array}\right\}\right] \\
& -\lim _{k \rightarrow \infty} \phi\left(p\left(z_{2 m_{k}-1}, z_{2 n_{k}}\right)+p\left(w_{2 m_{k}-1}, w_{2 n_{k}}\right)\right) \\
& =\psi\left(\frac{\epsilon}{2}\right)-\lim _{k \rightarrow \infty} \phi\left(p\left(z_{2 m_{k}-1}, z_{2 n_{k}}\right)+p\left(w_{2 m_{k}-1}, w_{2 n_{k}}\right)\right) \\
& =\psi\left(\frac{\epsilon}{2}\right)-\lim _{k \rightarrow \infty} \phi\left[\frac{1}{2}\left(\begin{array}{c}
p^{s}\left(z_{2 m_{k}-1}, z_{2 n_{k}}\right)+p\left(z_{2 m_{k}-1}, z_{2 m_{k}-1}\right) \\
+p\left(z_{2 n_{k}}, z_{2 n_{k}}\right)+p^{s}\left(w_{2 m_{k}-1}, w_{2 n_{k}}\right) \\
+p\left(w_{2 m_{k}-1}, w_{2 m_{k}-1}\right)+p\left(w_{2 n_{k}}, w_{2 n_{k}}\right)
\end{array}\right)\right] \\
& =\psi\left(\frac{\epsilon}{2}\right)-\lim _{t \rightarrow \frac{\epsilon}{2}} \phi(t) \text {, } \\
& \text { where } \frac{\epsilon}{2}=\lim _{k \rightarrow \infty} \frac{1}{2}\left(\begin{array}{c}
p^{s}\left(z_{2 m_{k}-1}, z_{2 n_{k}}\right)+p\left(z_{2 m_{k}-1}, z_{2 m_{k}-1}\right) \\
+p\left(z_{2 n_{k}}, z_{2 n_{k}}\right)+p^{s}\left(w_{2 m_{k}-1}, w_{2 n_{k}}\right) \\
+p\left(w_{2 m_{k}-1}, w_{2 m_{k}-1}\right)+p\left(w_{2 n_{k}}, w_{2 n_{k}}\right)
\end{array}\right) \\
& <\psi\left(\frac{\epsilon}{2}\right)
\end{aligned}
$$

which is a contradiction. Hence $\left\{z_{2 n}\right\}$ and $\left\{w_{2 n}\right\}$ are Cauchy sequences in the metric space $\left(X, p^{s}\right)$.

Letting $n, m \rightarrow \infty$ in

$$
\left|p^{s}\left(z_{2 n+1}, z_{2 m+1}\right)-p^{s}\left(z_{2 n}, z_{2 m}\right)\right| \leq p^{s}\left(z_{2 n+1}, z_{2 n}\right)+p^{s}\left(z_{2 m+1}, z_{2 m}\right) \text {. }
$$

we get

$$
\lim _{n \rightarrow \infty} p^{s}\left(z_{2 n+1}, z_{2 m+1}\right)=0
$$

Letting $n, m \rightarrow \infty$ in

$$
\left|p^{s}\left(w_{2 n+1}, w_{2 m+1}\right)-p^{s}\left(w_{2 n}, w_{2 m}\right)\right| \leq p^{s}\left(w_{2 n+1}, w_{2 n}\right)+p^{s}\left(w_{2 m+1}, w_{2 m}\right)
$$

we get

$$
\lim _{n \rightarrow \infty} p^{s}\left(w_{2 n+1}, w_{2 m+1}\right)=0 .
$$

Thus $\left\{z_{2 n+1}\right\}$ and $\left\{w_{2 n+1}\right\}$ are Cauchy sequences in the metric space $\left(X, p^{s}\right)$.

Hence $\left\{z_{n}\right\}$ and $\left\{w_{n}\right\}$ are Cauchy sequences in the metric space $\left(X, p^{s}\right)$.

Hence we have that $\lim _{n \rightarrow \infty} p^{s}\left(z_{n}, z_{m}\right)=0=\lim _{n \rightarrow \infty} p^{s}\left(w_{n}, w_{m}\right)$.

Now from definition of $p^{s}$ and from (2.5) we have

$$
\lim _{n \rightarrow \infty} p\left(z_{n}, z_{m}\right)=0
$$


and

$$
\lim _{n \rightarrow \infty} p\left(w_{n}, w_{m}\right)=0 \text {. }
$$

Suppose $f(X)$ is complete. Since $\left\{z_{2 n+1}\right\} \subseteq f(X)$ and $\left\{w_{2 n+1}\right\} \subseteq f(X)$ are Cauchy sequences in the complete metric space $\left(f(X), p^{s}\right)$, it follows that the sequences $\left\{z_{2 n+1}\right\}$ and $\left\{w_{2 n+1}\right\}$ are convergent in $\left(\mathrm{f}(\mathrm{X}), \mathrm{p}^{\mathrm{s}}\right)$. Thus

$$
\lim _{n \rightarrow \infty} p^{s}\left(z_{2 n+1}, u\right)=0
$$

and

$$
\lim _{n \rightarrow \infty} p^{s}\left(w_{2 n+1}, v\right)=0
$$

for some $u$ and $v$ in $f(X)$.

Since $u, v \in f(X)$. there exist $s, t \in X$ such that $u=f s$ and $v=f t$.

Since $\left\{z_{n}\right\}$ and $\left\{w_{n}\right\}$ are Cauchy sequences in $X$ and $\left\{z_{2 n+1}\right\} \rightarrow u$ and $\left\{w_{2 n+1}\right\} \rightarrow v$, it follows that $\left\{z_{2 n}\right\} \rightarrow u$ and $\left\{w_{2 n}\right\} \rightarrow v$.

From Lemma 1.1, we have

$$
p(u, u)=\lim _{n \rightarrow \infty} p\left(z_{2 n}, u\right)=\lim _{n \rightarrow \infty} p\left(z_{2 n+1}, u\right)=\lim _{n \rightarrow \infty} p\left(z_{n}, z_{m}\right)
$$

and

$$
p(v, v)=\lim _{n \rightarrow \infty} p\left(w_{2 n}, v\right)=\lim _{n \rightarrow \infty} p\left(w_{2 n+1}, v\right)=\lim _{n \rightarrow \infty} p\left(w_{n}, w_{m}\right)
$$

From (2.16), (2.17), (2.14) and (2.15) we have

$$
p(u, u)=0=p(v, v) .
$$

Now,

$$
\begin{aligned}
p(F(s, t), u) & \leq p\left(F(s, t), z_{2 n+1}\right)+p\left(z_{2 n+1}, u\right)-p\left(z_{2 n+1}, z_{2 n+1}\right) \\
& \leq p\left(F(s, t), G\left(x_{2 n+1}, y_{2 n+1}\right)\right)+p\left(z_{2 n+1}, u\right) .
\end{aligned}
$$

Therefore,

$$
\begin{aligned}
\psi(p(F(s, t), u)) \leq & \psi\left(p\left(F(s, t), G\left(x_{2 n+1}, y_{2 n+1}\right)\right)+p\left(z_{2 n+1}, u\right)\right) \\
\leq & \psi\left(p\left(F(s, t), G\left(x_{2 n+1}, y_{2 n+1}\right)\right)\right)+\psi\left(p\left(z_{2 n+1}, u\right)\right), \text { from }\left(\psi_{3}\right) \\
\leq & \frac{1}{2} \psi\left(p\left(u, z_{2 n}\right)+p\left(v, w_{2 n}\right)\right)- \\
& \phi\left(p\left(u, z_{2 n}\right)+p\left(v, w_{2 n}\right)\right)+\psi\left(p\left(z_{2 n+1}, u\right)\right) .
\end{aligned}
$$

Letting $n \rightarrow \infty$ and using (2.16), (2.17), (2.18) and $\left(\phi_{2}\right),\left(\psi_{1}\right)$ we get $\psi(p(F(s, t), u)) \leq 0$. Hence $F(s, t)=u=f s\left(\right.$ by $\left.\left(\psi_{2}\right)\right)$.

Similarly, we have $F(t, s)=v=f t$.

Since the pair $(F, f)$ is $w$ - compatible, we have $f u=F(u, v)$ and $f v=F(v, u)$. Suppose that $f u \neq u$ or $f v \neq v$.

$$
p^{s}\left(f u, z_{2 n}\right)=2 p\left(f u, z_{2 n}\right)-p(f u, f u)-p\left(z_{2 n}, z_{2 n}\right) .
$$


Letting $n \rightarrow \infty$, we get

$$
p^{s}(f u, u)=2 \lim _{n \rightarrow \infty} p\left(f u, z_{2 n}\right)-p(f u, f u)-0, \text { from }(2.5)
$$

or

$$
2 p(f u, u)-p(f u, f u)-p(u, u)=2 \lim _{n \rightarrow \infty} p\left(f u, z_{2 n}\right)-p(f u, f u)
$$

or

$$
p(f u, u)=\lim _{n \rightarrow \infty} p\left(f u, z_{2 n}\right), \text { from }(2.18) .
$$

Similarly, we have $p(f v, v)=\lim _{n \rightarrow \infty} p\left(f v, w_{2 n}\right)$.Thus

$$
\lim _{n \rightarrow \infty}\left[p\left(f v, z_{2 n}\right)+p\left(f v, w_{2 n}\right)\right]=p(f u, u)+p(f v, v)>0
$$

We have

$$
\begin{aligned}
p(f u, u) & \leq p\left(f u, z_{2 n+1}\right)+p\left(z_{2 n+1}, u\right)-p\left(z_{2 n+1}, z_{2 n+1}\right) \\
& \leq p\left(F(u, v), G\left(x_{2 n+1}, y_{2 n+1}\right)\right)+p\left(z_{2 n+1}, u\right)
\end{aligned}
$$

Thus,

$$
\begin{aligned}
\psi(p(f u, u)) \leq & \psi\left(p\left(F(u, v), G\left(x_{2 n+1}, y_{2 n+1}\right)\right)+\psi\left(p\left(z_{2 n+1}, u\right)\right), \text { from }\left(\psi_{3}\right)\right. \\
\leq & \frac{1}{2} \psi\left(p\left(f u, z_{2 n}\right)+p\left(f v, w_{2 n}\right)\right) \\
& -\phi\left(p\left(f u, z_{2 n}\right)+p\left(f v, w_{2 n}\right)\right)+\psi\left(p\left(z_{2 n+1}, u\right)\right)
\end{aligned}
$$

Similarly, we have

$$
\begin{aligned}
\psi(p(f v, v)) \leq & \frac{1}{2} \psi\left(p\left(f u, z_{2 n}\right)+p\left(f v, w_{2 n}\right)\right) \\
& -\phi\left(p\left(f u, z_{2 n}\right)+p\left(f v, w_{2 n}\right)\right)+\psi\left(p\left(w_{2 n+1}, v\right)\right) .
\end{aligned}
$$

Hence

$$
\begin{aligned}
\psi(p(f u, u)+p(f v, v)) \leq & \psi(p(f u, u))+\psi(p(f v, v)), \text { from }\left(\psi_{3}\right) \\
\leq & \psi\left(p\left(f u, z_{2 n}\right)+p\left(f v, w_{2 n}\right)\right) \\
& -2 \phi\left(p\left(f u, z_{2 n}\right)+p\left(f v, w_{2 n}\right)\right) \\
& +\psi\left(p\left(z_{2 n+1}, u\right)\right)+\psi\left(p\left(w_{2 n+1}, v\right)\right)
\end{aligned}
$$

Letting $n \rightarrow \infty$ and using $(2.19),\left(\phi_{1}\right),(2.16),(2.17)$ and $\left(\psi_{1}\right)$, we get

$$
\psi(p(f u, u)+p(f v, v))<\psi(p(f u, u)+p(f v, v))
$$

It is a contradiction. Hence $f u=u$ and $f v=v$. Thus

$$
F(u, v)=f u=u \text { and } F(v, u)=f v=v .
$$


Since $F(X \times X) \subseteq g(X)$, there exist $a, b \in X$ such that $u=F(u, v)=g a$ and $v=F(v, u)=g b$.

$$
\begin{aligned}
\psi(p(u, G(a, b))) & =\psi(p(F(u, v), G(a, b))) \\
& \leq \frac{1}{2} \psi(p(u, u)+p(v, v))-\phi(p(u, u)+p(v, v)) \\
& =\frac{1}{2} \psi(0)-\phi(0), \quad(\text { from }(2.18)) \\
& \leq 0, \quad(\text { since } \psi(0)=0 \text { and } \phi(0) \geq 0) .
\end{aligned}
$$

Hence $\psi(p(u, G(a, b)))=0$, which implies that $G(a, b)=u=g a$.

Similarly, we have $G(b, a)=v=g b$.

Since the pair $(\mathrm{G}, \mathrm{g})$ is $\boldsymbol{w}$ - compatible, we have $\mathrm{gu}=\mathrm{G}(\mathfrak{u}, v)$ and $\mathrm{g} v=\mathrm{G}(v, \mathfrak{u})$. Suppose $\mathrm{g} \mathfrak{u} \neq \mathfrak{u}$ or $g v \neq v$. We have

$$
\begin{aligned}
\psi(p(u, g u)) & =\psi(p(F(u, v), G(u, v))) \\
& \leq \frac{1}{2} \psi(p(u, g u)+p(v, g v))-\phi(p(u, g u)+p(v, g v))
\end{aligned}
$$

and

$$
\begin{aligned}
\psi(p(v, g v)) & =\psi(p(F(v, u), G(v, u))) \\
& \leq \frac{1}{2} \psi(p(u, g u)+p(v, g v))-\phi(p(u, g u)+p(v, g v))
\end{aligned}
$$

Hence

$$
\begin{aligned}
\psi(p(u, g u)+p(v, g v)) & \leq \psi(p(u, g u))+\psi(p(v, g v)) \\
& \leq \psi(p(u, g u)+p(v, g v))-2 \phi(p(u, g u)+p(v, g v)) \\
& <\psi(p(u, g u)+p(v, g v)) \quad(\text { since } \phi(t)>0 \forall t>0) .
\end{aligned}
$$

Hence $\mathfrak{g} u=u$ and $g v=v$.Thus,

$$
\mathrm{u}=\mathrm{gu}=\mathrm{G}(\mathrm{u}, v) \text { and } v=\mathrm{g} v=\mathrm{G}(v, \mathrm{u})
$$

From (2.20) and (2.21), it follows that $(u, v)$ is a common coupled fixed point of $F, G, f$ and $g$. Let $\left(u^{*}, v^{*}\right)$ be another common coupled fixed point of $F, G, f$ and $g$. We have

$$
\begin{aligned}
\psi\left(p\left(u, u^{*}\right)+p\left(v, v^{*}\right)\right) \leq & \psi\left(p\left(u, u^{*}\right)\right)+\psi\left(p\left(v, v^{*}\right)\right) \\
\leq & \psi\left(p\left(F(u, v), G\left(u^{*}, v^{*}\right)\right)\right)+\psi\left(p\left(F(v, u), G\left(v^{*}, u^{*}\right)\right)\right) \\
\leq & \frac{1}{2} \psi\left(p\left(u, u^{*}\right)+p\left(v, v^{*}\right)\right)-\phi\left(p\left(u, u^{*}\right)+p\left(v, v^{*}\right)\right) \\
& +\frac{1}{2} \psi\left(p\left(u, u^{*}\right)+p\left(v, v^{*}\right)\right)-\phi\left(p\left(u, u^{*}\right)+p\left(v, v^{*}\right)\right) \\
= & \psi\left(p\left(u, u^{*}\right)+p\left(v, v^{*}\right)\right)-2 \phi\left(p\left(u, u^{*}\right)+p\left(v, v^{*}\right)\right) \\
< & \psi\left(p\left(u, u^{*}\right)+p\left(v, v^{*}\right)\right)
\end{aligned}
$$


which is a contradiction. Hence $(u, v)$ is the unique common coupled fixed point of $F, G, f$ and $g$. Now we will show that $u=v$. Suppose $u \neq v$.

$$
\begin{aligned}
\psi(p(u, v)) & =\psi(p(F(u, v), G(u, v))) \\
& \leq \frac{1}{2} \psi(p(u, v)+p(v, u))-\phi(p(u, v)+p(v, u)) \\
& \leq \psi(p(u, v))-\phi(p(u, v)) \\
& <\psi(p(u, v)) .
\end{aligned}
$$

Hence $u=v$.

Thus $\mathfrak{u}=\mathfrak{f u}=\mathrm{F}(\mathfrak{u}, \mathfrak{u})=\mathrm{G}(\mathfrak{u}, \mathfrak{u})=\mathfrak{g u}$, that is, the common coupled fixed point of $F, G, f$ and $\mathfrak{g}$ has the form $(\mathfrak{u}, \mathfrak{u})$.

Received: June 2011. Revised: February 2012.

\section{References}

(1) Ishak Altun, Ferhan Sola and Hakan Simsek, Generalized contractions on partial metric spaces, Topology and its Applications, 157, (2010), 2778 - 2785.

(2) M.Abbas, M.Alikhan and S.Radenovic, Common coupled fixed point theorems in cone metric spaces for $w$ - compatible mappings, Applied Mathematics and Computation, Volume 217, Issue 1, (2010),195 - 202.

(3) T. Abdeljawad, E. Karapinar and K. Tas, Existence and uniqueness of a common fixed point on partial metric spaces, Appl. Math. Lett., Vol.24, Issue 11, (2011), 1900 - 1904.

(4) H. Aydi, Some coupled fixed point results on partial metric spaces, International Journal of Mathematics and Mathematical Sciences, Volume 2011, (2011), Article ID 647091.

(5) E. Karapinar and I.M. Erhan, Fixed point theorems for operators on partial metric spaces, Appl. Math. Lett., Vol.24, Issue 11, (2011), 1894 - 1899, doi:10.1016/j.aml.2011.05.013.

(6) M.Schellekens, The Smyth completion: a common foundation for denotational semantics and complexity analysis, Electronic Notes in Theoretical Computer Science, vol 1, (1995), 535 556.

(7) M.Schellekens, A characterization of partial metrizebility: domains are quantifiable, Theoretical Computer Sciences, vol 305, no. 1 - 3, (2003), 409 - 432.

(8) P.Waszkiewicz, Quantitative continuous domains, Applied Categorical Structures, vol 11, no. 1, (2003), $41-67$.

(9) P.Waszkiewicz, Partial metrizebility of continuous posets, Mathematical Structures in Computer Sciences, vol 16, no. 2, (2006), 359 - 372. 
(10) R. Heckmann, Approximation of metric spaces by partial metric spaces, Appl. Categ. Structures, no.1 - 2, 7, (1999), $71-83$.

(11) Sandra Oltra and Oscar Valero, Banach fixed point theorem for partial metric spaces, Rend.Istit.Mat.Univ.Trieste, vol XXXVI,(2004),17 - 26.

(12) Salvador Romaguera, A Kirk type characterization of completeness for partial metric spaces, Fixed Point Theory and Applications, vol.2010, (2010) Article ID 493298, 6 pages, doi:10.1155/2010/493298.

(13) S.G. Matthews, Partial metric topology,Proc. 8th Summer Conference on General Topology and Applications, in : Ann. New York Acad. Sci., vol. 728, (1994), 183 - 197.

(14) S.J. O'Neill, Partial metrics, valuations and domain theory, in : Proc. 11th Summer Conference on General Topology and Applications, vol 806 of Annals of the New York Academy of Sciences, (1996), $304-315$.

(15) S.Romaguera and M.Schellekens, Partial metric monoids and semi valuation spaces, Topology and Applications, vol 153, no.5 - 6, (2005), 948 - 962.

(16) S.Romaguera and O.Valero, A quantitative computational modal for complete partial metric space via formal balls, Mathematical Structures in Computer Sciences, vol.19, no.3, (2009), $541-563$.

(17) T.G.Bhaskar and V.Lakshmikantham, Fixed point theorems in partially ordered cone metric spaces and applications, Nonlinear Analysis: Theory, Methods and Applications, 65(7), (2006), $825-832$.

(18) Nguyen Van Luong and Nguyen Xuan Thuan, Coupled fixed points in partially order metric spaces and application, Nonlinear Analysis, 74, (2011), 983 - 992. 